\title{
A Novel Optimization Method of Optical Network Planning
}

\author{
Wu CHEN ${ }^{1, \text { a }}$ \\ ${ }^{1}$ The engineering \& technical college of chengdu university of technology, leshan, 614000 ,china; \\ awchen_leshan@126.com
}

Keywords:wavelength division multiplexing; optical network; network planning; network survivability

\begin{abstract}
In recent years, with the development of network technology and emergence of new Internet applications, the global Internet business has a trend of explosive growth, therefore, higher and higher requirements are raised on the bandwidth of the transmission network. Development of fiber optic transmission technology makes network transmission bandwidth be continuously increased; especially the wavelength division multiplexing (WDM) technology makes the telecom operators improve their network capacity by tens of times. The use of new technologies provides new subjects on network design and planning; in this paper, the optimization method for the backbone WDM transmission network planning is discussed as well as survivability issues in the network planning.
\end{abstract}

\section{Introduction}

With the development of data and multimedia services, the demand for long-distance transmission bandwidth increases rapidly, which imposes pressure on the fiber resources. The methods to enlarge the backbone network capacity based on the optical fiber communication mainly include Space Division Multiplexing (SDM), laying of new fiber optic cable, Time Division Multiplexing (TDM), increase of the transmission rate and Wavelength Division Multiplexing (WDM) / increasing the number of multiplexing wavelength. All WDMs mentioned in this paper refer to Dense WDM (DWDM). WDM is firstly proposed as a transmission technology, which is able to make full use of the huge fiber bandwidth resources to increase the transmission capacity of a piece of optical fiber by several times, tens of times or even hundreds of times compared with a single wavelength. Besides, each channel of WDM is transparent to data format, namely having nothing to do with the signaling rate and the electrical modulation mode. In network expansion and development, WDM is the ideal means of expansion and the convenient way to introduce new broadband services. New services or new capacity can be generated by increasing the wavelength.

\section{Intelligent (automatic) optical network}

Wavelength Division Multiplexing (WDM) is to converge the optical carrier signal (carrying a variety of information) with two or multiple different wavelengths at the transmitting terminal by means of the multiplexer (also known as combiner), which is coupled to the same fiber for transmission. At the receiving terminal, the de-multiplexer (also known as wave splitter or de-multiplexer) is utilized to separate optical carrier with various wavelengths, and then the optical receiver is used for further processing to restore the original signal. The technology to transmit two or numerous optical signals with different wavelengths in the same fiber is named Wavelength Division Multiplexing.

WDM is essentially the frequency division multiplexing (FDM) technology in the optical domain. Passageway of each wavelength is achieved through frequency-domain separation and occupies the bandwidth of a section of optical fiber. Wavelengths used in the WDM system is different, namely the specific standard wavelengths. In order to distinguish them from the common wavelengths of the SDH system, they are called the colored optical interface sometimes, while the interface of the common optical system is called "white light interface". 
If the communication system has different designs, the interval width between adjacent wavelengths varies. In accordance with different channel spacing, WDM can be subdivided into CWDM (Coarse Wavelength Division Multiplexing) and DWDM (Dense Wavelength Division Multiplexing). Channel spacing of CWDM is $20 \mathrm{~nm}$, but that of DWDM is from $0.2 \mathrm{~nm}$ to $1.2 \mathrm{~nm}$; therefore, CWDM is called the Coarse Wavelength Division Multiplexing technology compared with DWDM.

However, the WDM technology has its own characteristics. If telecom operators want to obtain good network performance at the lower cost of network construction and lay the foundation for the upgrading of the network in the future, these characteristics should be taken into account, which must be based on to implement reasonable planning. This introduces a problem about the transmission network planning.

\section{Network planning strategy}

In planning the transmission network, bandwidth demand for the different telecommunication services is assumed to be known. Bandwidth demand is acquired by forecasting and analyzing growth of various services. The purpose of the planning process is to draw up an optimized plan to meet the demand under certain technical and economic constraints. The ordinary tasks of the network planning are illustrated in Fig. 1:

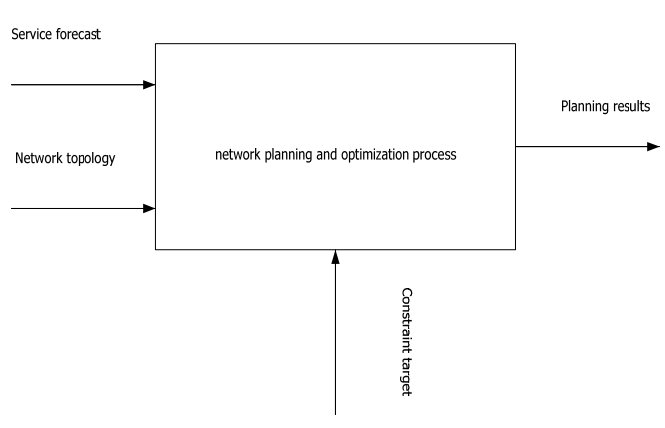

Fig. 1.Tasks of Network Planning

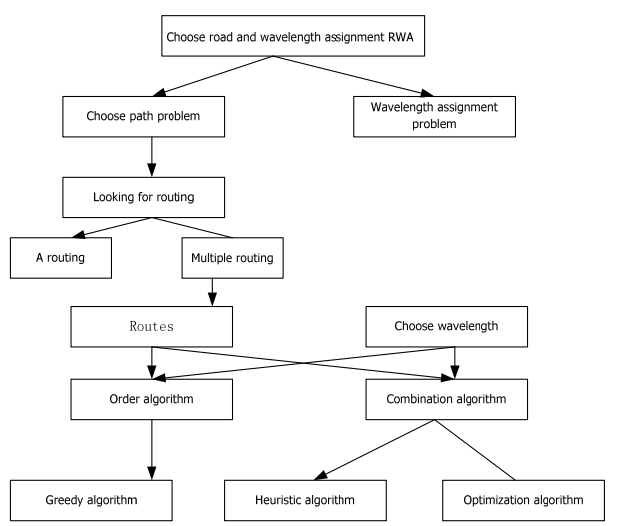

Fig. 2.RWA Algorithms Classification

Main inputs of the network planning process provided by the operators include the forecast of the service transmission demands during the planning period and the existing network structure built by the operators.

Optimization results of network planning will detail a variety of information including route used to achieve and restore service transmission request, resource allocation information (including working route and protecting / restoring the transmission route), network costs and resource utilization (fully enhance use of existing resources).

Transmission network planning is very complex, through which the integrated planning problems are usually be divided into smaller sub-problems by means of the decomposition method, thus reducing complexity of the integrated planning process; the decomposition method is considered to be the practical solution widely used in SDH transmission network. There are a lot of similarities between SDH and WDM networks, so the similar methods can be used to solve both of them. The main drawback of the decomposition method can be expressed as follows: it is difficult to control the planning problem in the case of more sub-problems, since the optimization depends not only on the efficiency of the algorithm to solve sub-problems but also on the harmonious relations of such sub-problems during the global optimization process under the condition.

In this paper, the planning process through the decomposition method is supplied by the operators, and the planning objective is to reduce the occupied resource required for transmission demands and lower the network cost from the operators. Because the planning is to implement route and wavelength allocation for the pre-determined service matrix, it belongs to the static RWA problem. The common solution algorithm is shown in Fig. 2:

When the static RWA problem is treated, the corresponding objectives are generally as follows: 
Reducing the number of wavelengths required to support the given optical path set----making full use of existing fiber; reducing the number of fiber required to support all services---making full use of existing wavelengths.

Static RWA results:

Wavelengths have been allocated for all optical path requests with the blocking rate of zero; all wavelengths are used up with a certain blocking rate, the capacity is required to be expanded. Starting from the demands of the planning algorithm, the physical topology and the logical topology of the network are pre-existing, so the optical cable should be pre-laid and the service matrix should be set by users (may be regarded as the survey result); for this reason, the corresponding objective is to support the given optical path set----making full use of existing fiber. If all optical path requests are satisfied in the largest range of available wavelengths, the less the number of actual opened wavelengths is, the less the corresponding devices are; if a certain blocking rate is still found to exist when all wavelengths are used up, the network needs expansion.

For the condition that the static RWA problem is decomposed into two sub-problems, route allocation and wavelength allocation, the algorithm of sequencing routing and wavelength allocation is presented as follows:

In this type of algorithm, the RWA problem is divided into two sub-problems, route allocation and wavelength allocation, which belongs to the sequential algorithm; on the one hand, the sequence should be selected, namely firstly selecting route and allocating wavelengths for a certain optical request; on the other hand, the criteria should be selected, which is based on to select route and wavelength for the optical path. The specific process is described as follows: a set of services are sequenced according to a certain strategy, then route and wavelengths are determined for these services in sequence; when the route and the wavelength are determined for a service, a set of available routes should be firstly determined for the service, which are sequenced as per a certain strategy, then wavelengths are allocated for the service on the available routes in sequence. During wavelength allocation, the wavelength allocation algorithm to solve the dynamic RWA problem is used. These algorithms are simple and require the shortest time; for the small-scale network, their performance is not much different from that of other algorithms, with more flexibility. The specific functional modules are divided as follows:

(1) Routing function module

Routing computation for a certain service in various service networks is achieved through the module.

A.Routing computation for a service based on a certain particular routing computation method, includes working route, protected route and restored route; during routing computation, route restrictions are required to be considered, such as the routing computation based on node uncorrelation, the routing computation based on link uncorrelation, the routing computation based on uncorrelation of the shared risk link group and so on.

B.The users may be allowed to modify manually computed service routing information and save the modified routing information according to the actual situation.

C.The loading function of new routing algorithm modules is provided. The software may be used to achieve dynamical loading of some new routing algorithm modules by means of research power of the laboratory.

D.The particular domain partitioning and routing functions in the ASON network may be achieved.

(2) Module of wavelength allocation

The resource allocation function for the services in different service networks can be achieved through the module.

A.Traditional resource allocation algorithms, such as the first-fit and the minimum load algorithms, are applied to realize resource allocation functions for the services in different service networks, including allocation of working resource, allocation of protected resource and allocation of restored resource; 
B.The loading function of new resource allocation algorithm modules is provided. The software may be used to achieve dynamical loading of some new resource allocation algorithm modules by means of research power of the laboratory.

\section{Network planning module.}

The network planning module is a special module and its intelligence is subject to certain restrictions. In the recent period, the positioning function of the network planning module is one of auxiliary planning and design tools, namely to design several schemes (network topology) by planning and design personnel according to relevant requirements; the network planning module is only utilized to make comparisons among these schemes and conFig. the optical path (see Fig. 3).

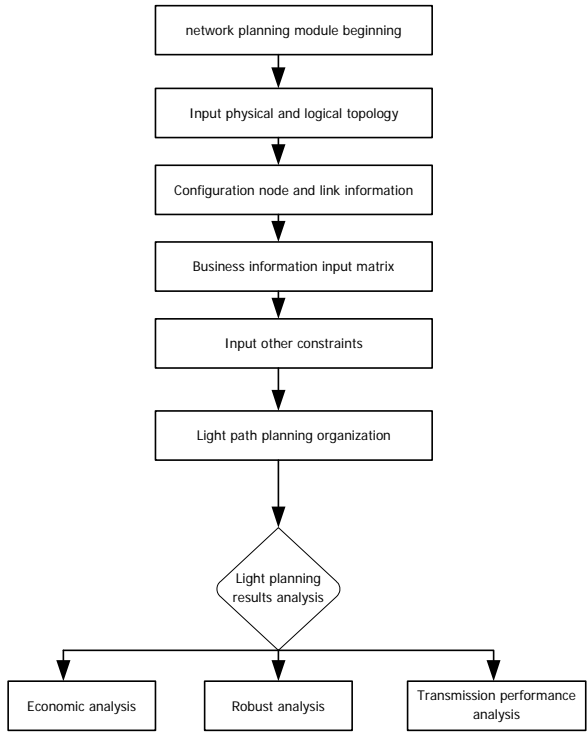

Fig.3.Flow Diagram of Network Planning Modules

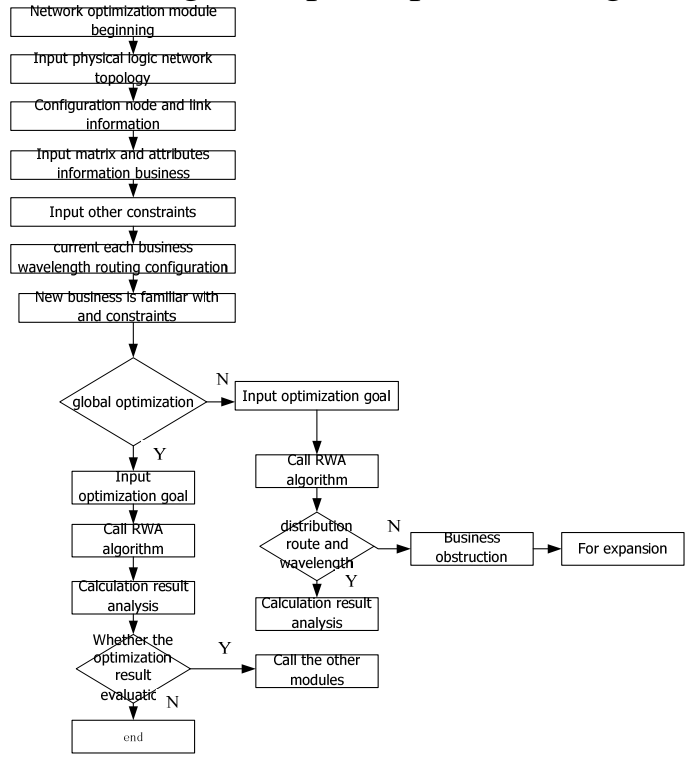

Fig.4.Flow Diagram of Network Optimization Modules

\section{Network optimization module.}

The main task of the network optimization module is to adjust the configuration during network operation, so as to make the network run under the optimized conditions and to improve utilization, quality and robustness of the network . Main applications scenarios include three points as follows:

For newly arrived services (or changed services), wavelength and routing allocation of the network may be required to be adjusted to meet the requirement for bandwidth and grade of service; at the moment, it is unnecessary to adjust wavelength and routing allocation for all services and the adjustment principle is to minimized the impact on the network service. Difference between the function and automatic end-to-end configuration function is that route and wavelength allocation for other services can be adjusted here, while the end-to-end configuration function fails. Because the services arrive in batches, the network management personnel must allocate wavelength and route as per relevant requirements (source node, destination node, bandwidth, grade of service etc.) for each arrived service; the process is generally completed manually or derived by means of a certain RWA algorithm. When the network operates for some time, the configuration of the entire network is not always optimal, such as low resource utilization, poor robustness (the service is excessively focused on some routes), high network blocking rate and so forth. Re-optimization may be performed by means of the RWA algorithm on the basis of service distribution at that time and the optimization objective. This process requires adjustment of all services (referring to the adjustable services, non-adjustable services may be reflected in the restrictions), and the optimization objective may be to maximize the resource utilization. Network performance obtained by means of different wavelength and route allocation algorithms is analyzed to optimize network route selection scheme, as shown in Fig. 4.

\section{Network survivability simulation module}

The so-called network survivability refers to the ability of the network to maintain an acceptable service quality grade during failure of the network and equipment. At present, more and more 
attention is paid to network survivability, protection and recovery of failure in improving the broadband network interconnection performance. Network failures can be divided into the hardware failure and the software failure. The former includes the node failure and the line failure, and fiber fracture is the major network failure in the optical fiber communication network, which will produce great effect on the service volume. In the software, the network survivability simulation function can be achieved from two following aspects. Different service grades are used to realize corresponding protection and recovery strategies; for example, for the service with the highest grade, " $1+1$ " exclusive protection is provided, " $1: 1$ " shared protection is provided for the service with the secondary grade, while the recovery strategy or the best-effort recovery strategy is provided for the service with the lowest grade. Moreover, for the sake of achievement of the network survivability simulation, it is assumed that a link or a node fails during network operation, and real-time recovery is implemented for the affected services as well as statistics of the restored performance.

\section{Conclusion}

The WDM optical network with route selection and wavelength allocation is considered to be one of the main directions of development of the next generation of high-speed wide-area backbone network. Static routing and wavelength allocation (RWA) in the optical network are to search the routes from the source node to the destination node and allocate wavelengths for the routes. On the other hand, the WDM technology enables the huge service volume to be centralized, with more and more remarkable network survivability issues. For the invulnerable optical networks with the path-protection strategy, the traditional RWA is transformed into such problems: the first is to seek for two physically separated paths for each service; the second is to allocate reasonable wavelengths (channels) for these paths. In the planning and optimization process of the optical network, the RWA algorithm integrating the survivability design will be repeatedly called by the topology planning and optimization steps, for this reason, it is the core in planning and optimizing the optical network. The objective to study the RWA problem is to minimize the number of the wavelengths and the blocking rate of the optical path.

\section{References}

[1] Special Issue on High-Capacity Optical Transport Networks, IEEE J.Select. Areas Commun., vol. 16, Sept. 1998.

[2] Special Issue on Optical Networks, IEEE J. Select. Areas Commun., vol.14, June 1996.

[3] Special Issue on Broadband Optical Networks, IEEE/OSA J. Lightwave Technol., vol. 11, May/June 1993.

[4] Special Issue on Dense Wavelength Division Multiplexing Techniques for High Capacity and Multiple Access Communication Systems, IEEE J. Select. Areas Commun, vol. 8, Aug. 1990.

[5] S. Melle, C. P. Pfistner, and F. Diner, "Amplifier and multiplexing technologies expand network capacity,” Lightwave Mag., pp. 42-46, Dec.1995.

[6] P. E. Green, “Optical networking update,” IEEE J. Select. Areas Commun., vol. 14, pp. 764-779, June 1996.

[7] C. Fan, "Keynote address-Optical networking: A paradigm shift," in WDM Forum, London, June 1998.

[8] B. Mukherjee, "WDM-based local lightwave networks_Part I:Single-hop systems," IEEE Network Mag., vol. 6, pp. 12-27, May .1992.

[9] “WDM-based local lightwave networks-Part II: Multihop systems,”IEEE Network Mag., vol. 6, pp. 20-32, July 1992. 